\title{
Flutuação populacional de Grapholita molesta (Busck) com armadilhas de feromônio sexual na cultura do pessegueiro em Bento Gonçalves, RS, Brasil
}

\author{
Seasonal fluctuation of Grapholita molesta (Busck) using sexual pheromon in \\ peach orchards in Bento Gonçalves, RS, Brazil
}

Cristiano João Arioli ${ }^{1}$ Geraldo Andrade Carvalho ${ }^{2}$ Marcos Botton ${ }^{3}$

RESUMO

A flutuação populacional de machos de Grapholita molesta (Busck, 1916) (Lepidoptera: Tortricidae) foi avaliada em dois pomares comerciais de pessegueiro da cultivar Chiripá em Bento Gonçalves, RS, entre julho de 2000 a junho de 2002. Os insetos foram capturados por meio de armadilhas Delta (duas por pomar) iscadas com a formulação comercial do feromônio sexual sintético (E-8-dodecenilacetato + Z-8-dodecenil-acetato + Z-8-dodecenol) e avaliados semanalmente. Foram observados quatro picos populacionais durante o período de produção do pessegueiro: quarta semana de agosto, primeira de novembro e dezembro, $e$ segunda de janeiro. A temperatura média dos sete dias anteriores à cada avaliação foi correlacionada positivamente com o número de adultos da G. molesta capturados nas armadilhas. As informações deste trabalho podem ser utilizadas para orientar os produtores de pessegueiro, visando a direcionar as táticas de controle para os períodos em que a população do inseto for mais elevada nos pomares comerciais.

Palavras-chave: Mariposa oriental, levantamento populacional, pêssego.

\section{ABSTRACT}

Seasonal fluctuation of male Grapholita molesta (Busck, 1916) (Lepidoptera: Tortricidae) was evaluated in two commercial peach orchards cv Chiripá in Bento Gonçalves, RS, from June of 2000 to July of 2002. The insects were evaluated weekly using Delta traps (two/orchard) baited with commercial synthetic sex pheromone (E-8-dodecenilacetato + Z-8-dodecenil-acetato + Z-8-dodecenol $)$. Four peaks were observed during peach production period: last week of August, first of November and December and second of January. Medium temperature seven days before evaluation was positively correlated with catches of male G. molesta in the traps. Information about seasonal fluctuation can be used for growers as a tool to guide insecticide treatments for pest control in peach orchards.

Key words: oriental fruit moth, seasonal fluctuation, peach.

\section{INTRODUÇÃO}

A cultura do pessegueiro ocupa uma área de 23.300 hectares no Brasil (SIDRA, 2002), sendo os Estados do Rio Grande do Sul, São Paulo, Santa Catarina, Paraná e Minas Gerais os maiores produtores (MARODIN \& SARTORI, 2000; SIDRA, 2002). No Rio Grande do Sul, principal produtor nacional, o pessegueiro é cultivado comercialmente em três pólos localizados em Pelotas, grande Porto Alegre e Serra Gaúcha que, juntos, somam cerca de 15.000 hectares (SIDRA, 2002). A cultura destaca-se por sua importância econômica e social, constituindo-se em uma alternativa para diversificação da matriz produtiva, absorção da mão-de-obra familiar e geração de renda às pequenas propriedades (SIDRA 2002; PROTAS \& MADAIL 2003,).

Dentre os fatores limitantes à exploração econômica do pessegueiro destaca-se a Mariposa Oriental Grapholita molesta (Busck) (Lepidoptera: Tortricidae) (BOTTON et al., 2001). Para o controle do

${ }^{1}$ Engenheiro Agrônomo, Doutorando em Fitossanidade, Universidade Federal de Pelotas (UFPel), CP 354, 96010-900, Pelotas, RS

${ }^{2}$ Engenheiro Agrônomo, Professor Doutor Entomologia, Universidade Federal de Lavras (UFL), CP 37, 37200-000, Lavras, MG.

${ }^{3}$ Engenheiro Agrônomo, Pesquisador Doutor Entomologia, Embrapa Uva e Vinho, CP 130, 95700-000, Bento Gonçalves, RS.

E-mail: marcos@cnpuv.embrapa.br. Autor para Correspondência. 
inseto, os produtores têm empregado inseticidas sintéticos seguindo um calendário pré-definido (a cada 10 a 15 dias) levando em consideração principalmente informações meteorológicas e o estádio de desenvolvimento da cultura, sem considerar a flutuação populacional da praga nos pomares (BOTTON et al., 2001). Este manejo utilizado pelos fruticultores, está se tornando cada vez mais inviável, visto que a sociedade passou a exigir frutas de qualidade, obtidas por meio de sistemas de produção que protejam o ambiente e a saúde dos trabalhadores e consumidores e que podem ser alcançados por meio da produção integrada (NORMAS, 2001).

O conhecimento da flutuação populacional da mariposa oriental na região de Bento Gonçalves permitirá definir o momento em que o inseto ocorre nos pomares e as variações no número de indivíduos ao longo do tempo, indicando qual o melhor momento para a utilização de táticas de controle (BERTOLDI, 1988; CARVALHO, 1990; GONZALES, 1993; NUÑES \& PAULLIER, 1995; BOTTON et al., 2001). Estudos visando conhecer a flutuação populacional dos adultos da $\boldsymbol{G}$. molesta nos pomares de pessegueiro foram conduzidos em diferentes regiões produtoras do Brasil (SILVEIRA NETO et al., 1981; REGINA \& MATIOLI, 1987; BERTOLDI, 1988; CARVALHO, 1990; HICKEL \& DUCROQUET, 1998; HICKEL, 2002). Com base nesses trabalhos, foi verificado que é possível direcionar os tratamentos para o controle da praga durante o ciclo de produção da cultura para os períodos em que a população apresentar níveis mais elevados nos pomares (SALLES, 1991). Além disso, é fator determinante para implantação de modelos matemáticos de previsão de ocorrência. Entretanto, devido à influência dos fatores ambientais, é importante que a população da praga seja conhecida nas diferentes regiões produtoras (SILVEIRA NETO et al., 1976).

Este trabalho foi realizado com o objetivo de se conhecer a flutuação populacional dos adultos da mariposa oriental em pomares comerciais de pessegueiro localizados na região da Serra Gaúcha, RS.

\section{MATERIAL E MÉTODOS}

A flutuação populacional dos adultos da G. molesta foi estudada no período de julho de 2000 a junho de 2002 em dois pomares de um hectare cada, da cultivar Chiripá, de ciclo tardio, implantados em 1990, no espaçamento $4 \mathrm{~m}$ x 6m, localizados no Distrito de Pinto Bandeira pertencente ao município de Bento Gonçalves, RS (latitude $29^{\circ} 07^{\prime}$ Sul, longitude 51 $26^{\circ}$ Oeste e altitude aproximada de 725 metros). As avaliações foram realizadas semanalmente, contando- se o número de machos capturados em armadilhas modelo Delta branca $(10 \mathrm{~cm}$ de altura x $19,5 \mathrm{~cm}$ de largura x $28,4 \mathrm{~cm}$ de comprimento e com área de fundo adesivo igual a $\left.385,3 \mathrm{~cm}^{2}\right)$ iscadas com o feromônio sexual sintético (E-8-dodecenil-acetato + Z-8-dodecenilacetato + Z-8-dodecenol), impregnado em septos de borracha (liberadores) na proporção de 95:5:1, fornecido pela Isca Tecnologias Ltda.

Em cada área experimental (pomar) foram instaladas duas armadilhas distanciadas no mínimo de $30 \mathrm{~m}$ entre si, posicionadas nas plantas numa altura de $1,7 \mathrm{~m}$ do solo (BERTOLDI 1988). Os septos foram substituídos a cada seis semanas e o fundo contendo substância adesiva, sempre que verificada a perda de adesividade. Em cada pomar, aplicaram-se os tratamentos fitossanitários, conforme o calendário estabelecido pelos fruticultores.

A flutuação populacional de adultos foi demonstrada graficamente em cada ano plotando-se o número médio de machos/armadilha/semana em função do tempo e calculando-se o erro padrão da média (quatro armadilhas). Foram feitas correlações simples (teste de correlações de Pearson) entre o número médio de adultos da G. molesta capturados semanalmente versus a temperatura média diária $\left({ }^{\circ} \mathrm{C}\right)$ e a precipitação pluviométrica $(\mathrm{mm})$ acumulada registradas nos sete dias anteriores à avaliação. Os dados climáticos foram obtidos em uma estação meteorológica localizada a distância de 1.500 metros das áreas experimentais.

\section{RESULTADOS E DISCUSSÃO}

Foram observados quatro picos populacionais distintos da mariposa oriental durante $o$ período de produção do pessegueiro (agosto a janeiro) nas duas safras agrícolas (Figuras 1 e 2). A presença dos adultos nos pomares foi verificada a partir do mês de julho; no entanto, o máximo de captura foi observado na metade do mês de agosto (Figuras 1 e 2). A captura observada em meados de agosto foi proveniente dos insetos que passaram o inverno em diapausa na forma de lagarta de 3 o ínstar, visto que não foram obtidas capturas significativas de adultos entre os meses de maio a julho nos dois anos de avaliação (Figuras 1 e 2).

O biofix da G. molesta (momento em que as capturas da praga nas armadilhas de feromônio sexual passam a ser constantes) ocorreu nos pomares na primeira semana de agosto nos dois anos de avaliação. Esta informação é importante para trabalhos de validação de modelos de previsão de ocorrência da G. molesta com base nas exigências térmicas (RICE et al., 1982; GRELLMANN et al, 1992; HICKEL, 2002).

Ciência Rural, v.35, n.1, jan-fev, 2005. 


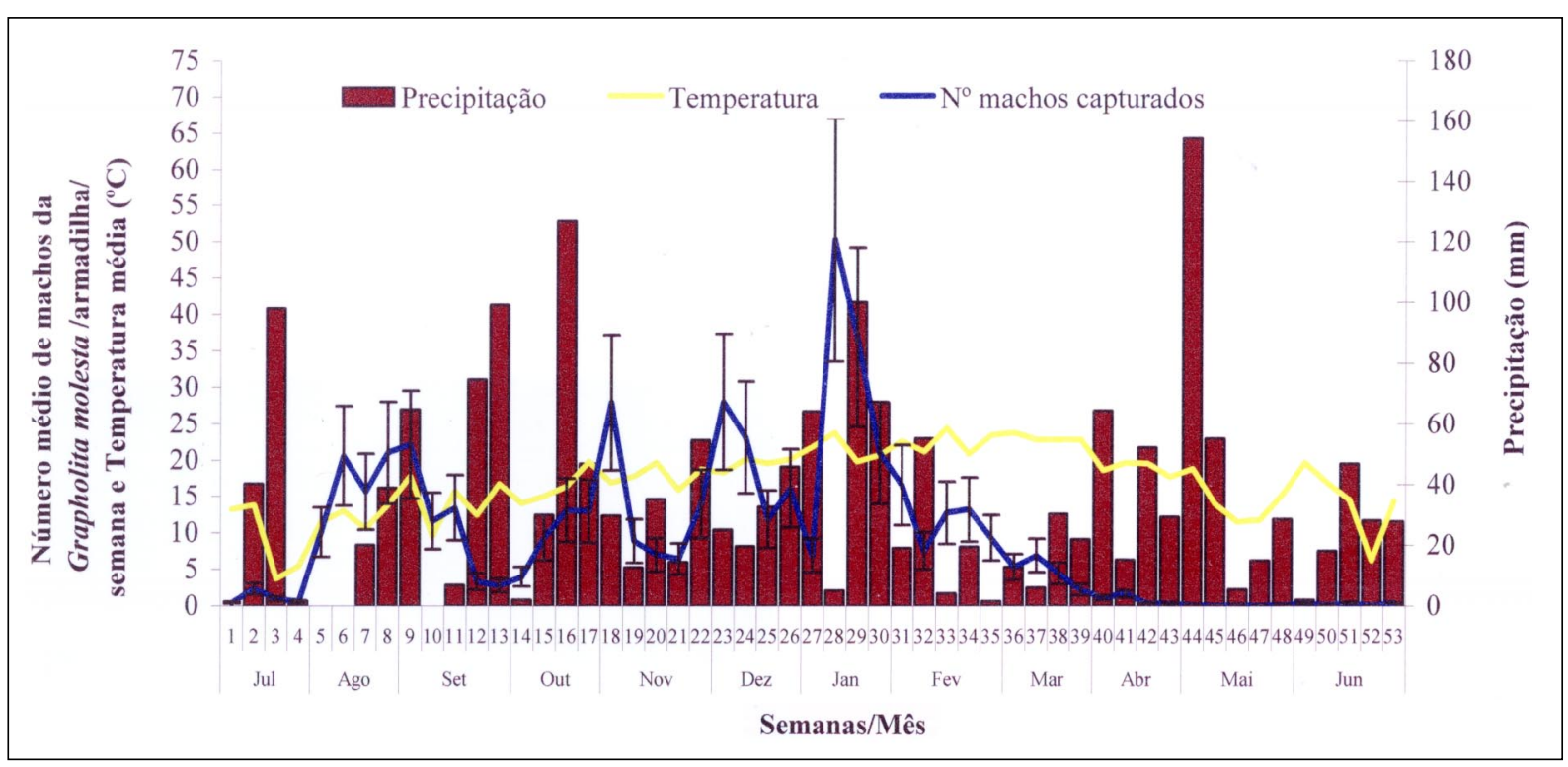

Figura 1 - Flutuação populacional de machos adultos da Grapholita molesta, capturados em armadilhas iscadas com feromônio sexual sintético, em pomares de pessegueiro da cultivar Chiripá. Os valores de temperatura média e precipitação pluviométrica obtidos sete dias anteriores às avaliações no período de Julho de 2000 a Junho de 2001, foram acumulados. Bento Gonçalves, RS.

Os adultos provenientes das lagartas que passaram o inverno em diapausa deram origem à primeira geração que se desenvolveu sobre a cultura do pessegueiro na primavera, apresentando máxima atividade de vôo entre o final de outubro e início de novembro (Figuras 1 e 2). O pico de vôo dos adultos da segunda geração foi observado entre o final de novembro e início de dezembro (Figuras 1 e 2). Os adultos da terceira geração apresentaram acme de captura em meados de janeiro (Figuras 1 e 2). Esta geração caracterizou-se por resultar na maior captura nos dois anos de avaliação. Os indivíduos da quarta geração apresentaram predominância de atividade em meados de fevereiro, porém, tanto este pico populacional como os que ocorreram em março e abril não foram facilmente caracterizados provavelmente devido à sobreposição de gerações (Figuras 1 e 2).

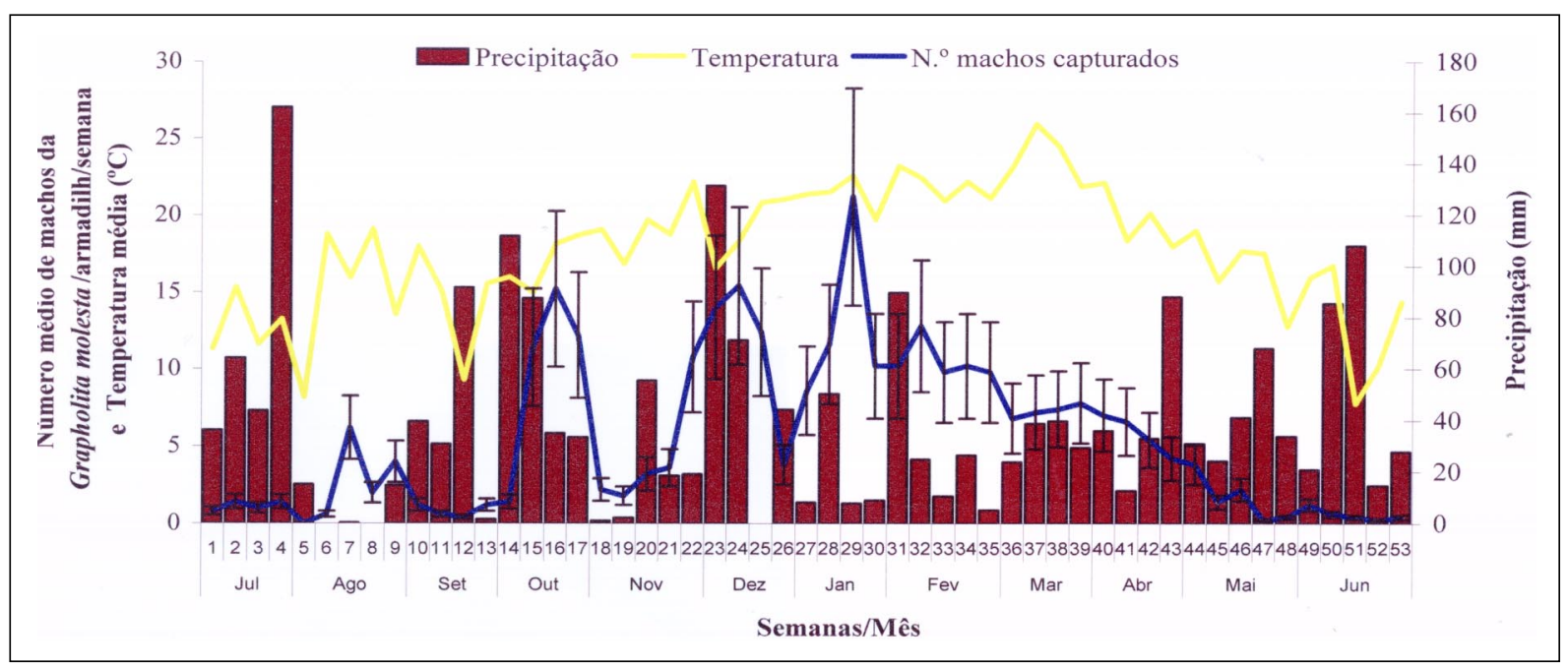

Figura 2 - Flutuação populacional de machos adultos da Grapholita molesta, capturados em armadilhas iscadas com feromônio sexual sintético, em pomares de pessegueiro da cultivar Chiripá. Os valores de temperatura média e precipitação pluviométrica obtidos sete dias anteriores às avaliações no período de Julho de 2001 a Junho de 2002, foram acumulados. Bento Gonçalves, 
Considerando a temperatura base de $8,99^{\circ} \mathrm{C}$ e a constante térmica de 482GD (Graus-dia) determinados por GRELLMANN et al., (1992) e o biofix no início de agosto, seriam estimadas quatro gerações da praga durante a safra do pessegueiro na Região da Serra Gaúcha. Estas gerações são caracterizadas pelos vôos que ocorrem em meados de agosto, final de outubro, final de novembro e meados de janeiro (Figuras 1 e 2). Com base nas exigências térmicas do inseto e a temperatura média diária obtida nas duas safras avaliadas, estimou-se a ocorrência de seis gerações da mariposa oriental durante o ano. As gerações que ocorreram após a colheita do pessegueiro foram monitoradas mediante os vôos a partir de fevereiro, registrando-se menores capturas de machos nas armadilhas de feromônio, provavelmente, devido à indisponibilidade de alimento (lignificação dos ponteiros), redução da temperatura e do fotoperíodo.

De maneira geral, os picos populacionais observados no período de agosto a janeiro são semelhantes aos verificados por BERTOLDI (1988) e CARVALHO (1990) na região de Pelotas, RS e HICKEL \& DUCROQUET (1998) em Videira, SC. De acordo com NUÑES \& PAULLIER (1995), nas condições da zona Sul do Uruguai, a mariposa oriental apresenta cinco gerações anuais, sendo que, em alguns anos, uma sexta geração parcial é observada, o que também é constatado no Chile (GONZALES 1986) e Argentina (REISING, 2003, Informe verbal). Segundo esses autores, os picos populacionais de G. molesta no Uruguai, Chile e Argentina ocorrem em meados de setembro e de novembro, início de janeiro, meados de fevereiro e início de março, respectivamente.

Através da análise de correlação simples dos fatores climáticos referentes aos sete dias anteriores à cada contagem do número de adultos, observou-se que a temperatura média diária foi o fator climático que exerceu maior influência, correlacionando-se positivamente com o número de insetos capturados nas armadilhas (Tabela 1), concordando com os resultados obtidos por HICKEL (2002). Assim, ocorreram maiores capturas com o incremento da temperatura (Figuras 1 e 2). Este fator também pôde ser observado a partir do pico populacional de dezembro, quando, devido ao aumento da temperatura, o intervalo de tempo entre os picos populacionais foi mais curto (Figuras 1 e 2). Não foi obtida correlação positiva entre a precipitação acumulada sete dias antes das avaliações e o número de indivíduos capturados nas armadilhas (Tabela 1).
Com relação aos danos ocasionados pelo inseto, embora este parâmetro não tenha sido quantificado neste trabalho, observou-se que as lagartas provenientes da geração pós-diapausa (agosto) não danificam as cultivares de pessegueiro de ciclo tardio, como é o caso do Chiripá, porque, nesse período, as lagartas não encontram ponteiros disponíveis para se desenvolver. Este fato foi observado no Uruguai, onde somente a partir da segunda geração da praga (novembro) ocorrem ataques significativos aos ponteiros (até 40\%) e frutos (3\%) (NUÑES \& PAULLIER, 1995). Os maiores prejuízos são provocados pela terceira geração de lagartas, pois ocorre um incremento no ataque aos frutos, com perdas entre $30 \%$ a $50 \%$ (NUÑES \& PAULLIER, 1995).

Embora a primeira geração (geração de primavera) da G. molesta não seja considerada de risco para cultivares de ciclo tardio (NUÑES \& PAULLIER, 1995; BOTTON et al., 2001), a determinação do momento em que as capturas da praga nas armadilhas de feromônio sexual passam a ser constantes (biofix) nas diferentes regiões, é de fundamental importância para o emprego de modelos matemáticos de previsão de ocorrência que usam como base as exigências térmicas (RICE et al., 1982; GRELLMANN et al., 1992; HICKEL, 2002).

As informações obtidas no município de Bento Gonçalves, a princípio, podem ser utilizadas para orientar os fruticultores da região da Serra Gaúcha no controle da mariposa oriental de acordo com os picos populacionais que ocorrem durante o ciclo de produção do pessegueiro. No entanto, como os fatores climáticos são variáveis anualmente e entre os diferentes microclimas, é fundamental que a população do inseto seja monitorada em cada pomar e sejam conduzidos estudos visando associar o nível populacional de adultos capturados nas armadilhas e o dano causado pelas lagartas nas diferentes gerações.

Tabela 1 - Correlação entre o número médio de machos da Grapholita molesta capturados em armadilhas de feromônio sexual, durante as safras 2000/2001 e 2001/2002, temperatura média diária $\left({ }^{\circ} \mathrm{C}\right)$ e precipitação acumulada (mm) nos sete dias anteriores à cada avaliação. Bento Gonçalves, RS.

\begin{tabular}{lcc}
\hline $\begin{array}{c}\text { № médio de } \\
\text { machos capturados }\end{array}$ & $\begin{array}{c}\text { Temperatura média } \\
\text { diária }\left({ }^{\circ} \mathrm{C}\right)\end{array}$ & $\begin{array}{c}\text { Precipitação } \\
\text { acumulada }(\mathrm{mm})\end{array}$ \\
\hline Safra 2000/2001 & $0,31092^{* *}$ & $-0,0149^{\mathrm{ns}}$ \\
Safra 2001/2002 & $0,59150^{* *}$ & $-0,07058^{\mathrm{ns}}$ \\
\hline
\end{tabular}

** Significativo a $1 \%$ de probabilidade de erro pelo teste $\mathrm{t}$

${ }^{\text {ns }}$ não significativo a $1 \%$ de probabilidade de erro pelo teste $t$.

Ciência Rural, v.35, n.1, jan-fev, 2005. 


\section{CONCLUSÕES}

Em Bento Gonçalves, RS, são observados quatro picos populacionais de adultos da Grapholita molesta durante o período de produção (agosto a janeiro) do pessegueiro da cultivar Chiripá. A temperatura média de sete dias anteriores às avaliações influencia a captura dos machos da Mariposa Oriental em armadilhas de monitoramento contendo feromônio sexual sintético. A precipitação acumulada nos sete dias antes das avaliações não influencia o número de indivíduos capturados nas armadilhas.

\section{AGRADECIMENTOS}

À Isca Tecnologias Ltda, pelo fornecimento dos feromônios sexuais e armadilhas de captura, e aos produtores de pêssego Genésio Contini e Ênio Provenci, pela utilização dos pomares para a realização deste trabalho. Ao bolsista de estágio técnico da Fapergs, Odimar Z. Zanardi, pelo auxílio durante a condução do experimento.

\section{INFORME VERBAL}

Cristina Reising. Laboratório de Entomologia Agrícola da Estação Experimental Agropecuária do INTA Mendoza - Argentina. E-mail: reising@arnet.com.br

\section{REFERÊNCIAS BIBLIOGRÁFICAS}

BERTOLDI, L.H.M. Flutuação populacional de Grapholita molesta (Busck, 1916) (Lepidoptera: Tortricidae) e alguns aspectos de sua biologia em pomar de pessegueiro. 1988. 48f. Dissertação (Mestrado em Agronomia) - Curso de Pós graduação em Agronomia, Universidade Federal de Pelotas.

BOTTON, M. et al. Monitoramento da mariposa oriental Grapholita molesta (Busck, 1916) na cultura do pessegueiro. Bento Gonçalves : Embrapa - CNPUV, 2001. 4p. (Embrapa CNPUV. Comunicado Técnico, 38).

CARVALHO, R.P.L. Manejo integrado de pragas do pessegueiro In: CROCOMO, W.B. (Org.). Manejo integrado de pragas. São Paulo : UNESP, 1990. Cap.16, p.323-358.

GONZALES, R.H. Fenologia de la polilla oriental del durazno. Aconex, Santiago, n.12, p.5-12, 1986.

GONZALES. R.H. Sistemas de monitoreo y manejo de las polillas da fruta (Cydia molesta y $C$. pomonela). Santiago : Universidad de Chile, 1993. 60p.

GRELLMANN, E.O. et al. Necessidades térmicas e estimativa do número de gerações de Grapholita molesta (Busck, 1916) (Lepidoptera: Olethreutidae) em Pelotas, RS. Pesquisa Agropecuária Brasileira, Brasília, v.27, n.7, p.999-1004, 1992.

HICKEL, E.R. Flutuação populacional e previsão da atividade de vôo da Grapholita molesta (Busck, 1916) (Lepidoptera: Tortricidae) em pomares de pessegueiro e ameixeira. 2002. 86f. Tese (Doutorado em Entomologia) Curso de Pós-graduação em Entomologia, Universidade Federal de Viçosa.

HICKEL, E.R.; DUCROQUET, J.H.J. Monitoramento e controle da grafolita ou mariposa oriental no Alto Vale do Rio do Peixe. Agropecuária Catarinense, Florianópolis, v.11, n.2, p.8-11, 1998.

MARODIN, G.A.B.; SARTORI, I.A. Situação das frutas de caroço no Brasil e no mundo. In: SIMPÓSIO INTERNACIONAL DE FRUTAS DE CAROÇO PÊSSEGOS, NECTARINAS E AMEIXAS, 1., 2000, Porto Alegre. Anais... Porto Alegre : UFRGS, 2000. p.7-16.

NORMAS de produção integrada de pêssego (PIP): versão II. Pelotas : UFPel: Embrapa, UFRGS, URCAMP, 2001. 52p.

NUÑES, S.; PAULlIER, J. Cydia molesta (Busck). In: BENTANCOURT, C.M.; SCATONI, I.B. Lepidopteros de importancia economica. Reconocimiento, biologia y daños de las plagas agrícolas y florestales. Uruguai : Agropecuaria Hemisferio Sur, 1995. p.32-40,.

PROTAS, J.F.da. S.; MADAIL, J.C.M. Características econômicas e sociais da produção de pêssego no Rio Grande do Sul. In GARRIDO, L.T.; BOTTON, M. (Ed). Sistema de produção de pêssego de mesa na região da Serra Gaúcha. Capturado em 19 set. 2003. Online. Disponível em http://sistemasdeproducao. cnptia.embrapa.br/ FontesHTML/Pessego/PessegodeMesa RegiaoSerraGaucha/ pragas.htm.

REGINA, M.A.; MATIOLI, J.C. Flutuação populacional de Grapholita molesta (Busk) (Lep.: Olethreutidae) em pessegueiros no Sul de Minas Gerais. In:CONGRESSO BRASILEIRO DE ENTOMOLOGIA, 11., 1987, Campinas, SP. Anais... Campinas: IAC, 1987. V.11, p.140.

RICE, R.E. et al. Monitoring and modeling oriental fruit moth in California. California Agriculture, Bakland, v.6, n.1/2, p.11$12,1982$.

SALLES, L.A.B. de. Grafolita (Grapholita molesta): Biologia e controle. Pelotas : Embrapa - CNPFT, 1991. 13p. (Embrapa - CNPFT. DOCUMENTOS, 42).

SALLES, L.A.B. de. Principais pragas e seu controle. In: MEDEIROS, C.A.B.; RASEIRA, M. do C. A cultura do pessegueiro. Brasília : EMBRAPA- CPACT, 1998. Cap.8, p.206-242.

SIDRA - Sistema IBGE de Recuperação Automática. Desenvolvido pelo Instituto Brasileiro de Geografia e Estatística. Apresenta informações estatísticas. Capturado em 11/dez/2002. Online. Disponível em: <http:/ /www.sidra.ibge.gov.br/>.

SILVEIRA NETO, S. et al. Manual de ecologia dos insetos. São Paulo : Agronômica Ceres, 1976. 420p.

SILVEIRA NETO, S. et al. Flutuação populacional de Grapholita molesta (Busck) (Lepidoptera: Olethreutidae) em pessegueiro e nectarina, com o uso de feromônio sexual sintético. Anais da Sociedade Entomológica do Brasil, Londrina, v.10, n.1, p.43-49, 1981. 